\title{
Article \\ Limitation of Screening of Different Variants of SARS-CoV-2 by RT-PCR
}

\author{
Agathe Boudet $^{1, *}$, Robin Stephan ${ }^{2}$, Sophie Bravo ${ }^{3}$, Milène Sasso ${ }^{2,4}$ and Jean-Philippe Lavigne ${ }^{1} \mathbb{C}$ \\ 1 Virulence Bactérienne et Infections Chroniques, INSERM U1047, Université Montpellier, 30000 Nîmes, France; \\ jean.philippe.lavigne@chu-nimes.fr \\ 2 Department of Microbiology and Hospital Hygiene, CHU Nîmes, 30000 Nîmes, France; \\ robin.stephan@chu-nimes.fr (R.S.); milene.sasso@chu-nimes.fr (M.S.) \\ 3 Department of Biochemistry, CHU Nîmes, 30000 Nîmes, France; sophie.bravo@chu-nimes.fr \\ 4 MIVEGEC, IRD, CNRS, Université Montpellier, 34000 Montpellier, France \\ * Correspondence: agathe.boudet@chu-nimes.fr; Tel.: +33-466-683-202
}

Citation: Boudet, A.; Stephan, R.; Bravo, S.; Sasso, M.; Lavigne, J.-P. Limitation of Screening of Different Variants of SARS-CoV-2 by RT-PCR Diagnostics 2021, 11, 1241. https:// doi.org/10.3390/diagnostics11071241

Academic Editor: Renato Millioni

Received: 9 June 2021

Accepted: 8 July 2021

Published: 12 July 2021

Publisher's Note: MDPI stays neutral with regard to jurisdictional claims in published maps and institutional affiliations.

Copyright: () 2021 by the authors. Licensee MDPI, Basel, Switzerland. This article is an open access article distributed under the terms and conditions of the Creative Commons Attribution (CC BY) license (https:// creativecommons.org/licenses/by/ $4.0 /)$.

\begin{abstract}
Since January 2021, the diffusion of the most propagated SARS-CoV-2 variants in France (UK variant 20I/501Y.V1 (lineage B.1.1.7), 20H/H501Y.V2 (lineage B.1.351) and 20J /H501Y.V3 (lineage P.1)) were urgently screened, needing a surveillance with an RT-PCR screening assay. In this study, we evaluated one RT-PCR kit for this screening (ID SARS-CoV-2/UK/SA Variant Triplex ${ }^{\circledR}$, ID Solutions, Grabels, France) on 2207 nasopharyngeal samples that were positive for SARS-CoV-2. Using ID Solutions kit, 4.1\% (92/2207) of samples were suspected to belonged to B.1.351 or P.1 variants. Nextgeneration sequencing that was performed on $67.4 \%(62 / 92)$ of these samples confirmed the presence of a B.1.351 variant in only $75.8 \%$ of the samples $(47 / 62)$. Thirteen samples belonged to the UK variant (B.1.1.7), and two to A.27 with N501Y mutation. The thirteen with the UK variant presented one mutation in the S-gene, near the $\Delta \mathrm{H} 69 / \Delta \mathrm{V} 70$ deletion (S71F or A67S), which impacted the detection of $\Delta \mathrm{H} 69 / \Delta \mathrm{V} 70$ deletion. Using another screening kit (PKampVariantDetect SARS-CoV-2 RT-PCR combination 1 and $3^{\circledR}$ PerkinElmer, Waltham, MA, USA) on the misidentified samples, we observed that the two mutations, S71F or A67S, did not impact the detection of the UK variant. In conclusion, this study highlights the limitations of the screening strategy based on the detection of few mutations/deletions as well as it not being able to follow the virus evolution.
\end{abstract}

Keywords: SARS-CoV-2; RT-PCR; screening method; variants

\section{Introduction}

Since December 2019, a pandemic linked to a severe acute respiratory syndrome coronavirus 2 (SARS-CoV-2) caused pneumonia and severe acute respiratory syndrome worldwide [1,2]. From September 2020, new variants of concern (VOC) of SARS-CoV-2 were detected in Europe [3]. The first was the United Kingdom variant 20I/501Y.V1 (lineage B.1.1.7) [4], followed by two other lineages: the South-African variant 20H/H501Y.V2 (lineage B.1.351) [5] and the Brazilian variant 20J/H501Y.V3 (lineage P.1) [6,7]. Recently, other variants have emerged, such as the SARS-CoV-2 lineage A.27, which was firstly identified in Denmark in December 2020 but has its first origin in France [8]. Since January 2021, the propagation of these variants in France required an increased surveillance, based on RT-PCR screening assay, due to their important transmissibility and their potential to evade the host immune system [9]. Two of these lineages, B.1.351 and P.1, were of specific concern because they harbored the E484K mutation, which has been shown to enhance escape from neutralizing antibody inhibition in vitro [10], and may be associated with a reduced efficacy of the vaccine [11,12]. The aim of this study was to evaluate the main RT-PCR kit that is used for this screening: the ID SARS-CoV-2/UK/SA Variant Triplex ${ }^{\circledR}$ (ID Solutions, Grabels, France). 


\section{Materials and Methods}

\subsection{Samples}

This study was conducted at Nîmes University Hospital (France) between 27 January and 30 April 2021, commencing from the first nasopharyngeal screening performed. All admitted patients in our hospital were tested when they presented with potential COVID-19 related symptoms, including: fever, persistent cough, fatigue, myalgia, shortness of breath, diarrhoea, abdominal pain, chest pain, sore throat, loss of smell or taste; or when they were in close or prolonged contact with confirmed COVID-19 infected patients. For each patient, a nasopharyngeal swab was collected and sent to the Department of Microbiology that is accredited to perform RT-PCR assay for SARS-CoV-2. This retrospective study was approved by the local institutional review boards of the Nîmes University Hospital, France (IRB number: 20.05.01, approved on 4 May 2020). During this routine sample, we obtained a non-opposed consent of the patients to participate in SARS-CoV-2 studies. No data concerning patients and no follow-up were collected.

\subsection{RT-PCR SARS-CoV-2 and Variants Detection}

RNA was extracted from clinical samples using the chemagic viral DNA/RNA kit special 96 on the chemagic platform (PerkinElmer, Waltham, MA, USA). The RT-PCR was performed using the kit SARS-CoV-2 R-Gene ${ }^{\circledR}$ (bioMérieux, Marcy-l'Étoile, France), following the manufacturer's recommendations. After this detection of the presence/absence of the virus, we screened the main variants that are circulating in Europe on positive specimens with cycle threshold $(\mathrm{Ct})$ values lower than 35 . This subsequent screening was performed with the ID SARS-CoV-2/UK/SA Variant Triplex ${ }^{\circledR}$ kit (ID Solution, Grabels, France) [13]. This kit contained a positive control and a reaction mix with reverse transcriptase, Taq polymerase, primers and hydrolysis probes for the detection of SARS-CoV-2 targets, VOC202012/01 and 501Y.V2 variants. Assays were carried out with the following running conditions: $50{ }^{\circ} \mathrm{C}$ for $10 \mathrm{~min}$, followed by $2 \mathrm{~min}$ at $95^{\circ} \mathrm{C}, 40$ cycles of $95^{\circ} \mathrm{C}$ for $10 \mathrm{~s}$ and $65^{\circ} \mathrm{C}$ for $30 \mathrm{~s}$, according to manufacturer's recommendations. Another screening kit (PKampVariantDetect SARS-CoV-2 RT-PCR combination 1 and $3^{\circledR}$ (PerkinElmer)) was also performed retrospectively on the positive samples which belonged to non-UK variants, using the ID Solution kit [14]. All of these RT-PCR were conducted according to manufacturer's instructions on the QuantStudio5 thermocycler (ThermoFisher Scientific, Waltham, MA, USA). The two screening kits presented a similar universal target of SARS-CoV-2 (N/ORF1/ab-gene), and two targets on the S-gene, the target of six nucleotides deletions $(\Delta \mathrm{H} 69 / \Delta \mathrm{V} 70)$ and the N501Y mutation. The Perkin Elmer RT-PCR kit had one more target for the E484K mutation, also on the S-gene. According to the kit used, three or four positive signals were present in the UK variant (Figure 1). Two or three positive targets that were composed by the universal target SARS-CoV-2 and the N501Y mutation + / - the E484K mutation suggested the presence of the variant 20H/501Y.V2 (B.1.351) or 20J/501Y.V3 (P.1). If the deletion of $\Delta \mathrm{H} 69 / \Delta \mathrm{V} 70$ only was observed, the contamination might be due to a wild-type strain with a deletion, or another variant. The absence of the different variant targets $(\Delta \mathrm{H} 69 / \Delta \mathrm{V} 70, \mathrm{~N} 501 \mathrm{Y}, \mathrm{E} 484 \mathrm{~K})$ was detected by the presence of the universal target of SARS-CoV-2 only and corresponded to a wild-type strain.

\subsection{NGS Sequencing}

Next generation sequencing (NGS) was performed on samples with a suspicion of B.1.351 and P.1 when Ct values were $<30$ on the first RT-PCR SARS-CoV-2 R-Gene ${ }^{\circledR}$. Library preparation was performed with Ion AmpliSeq ${ }^{\mathrm{TM}}$ Library Kit Plus $^{\circledR}$ and Ion Torrent ${ }^{\mathrm{TM}}$ Dual Barcode Kit 1-96, according to the manufacturer's recommendations (Ion torrent, ThermoFisher Scientific) [15]. Ion library Taqman quantitation kit ${ }^{\circledR}$ was used to quantify and normalize the library. Sample libraries were pooled and processed using IonTorrent platform Ion S5 ${ }^{\mathrm{TM}}$. CLC genomics (Qiagen, Hilden, Germany) was used for bioinformatics analysis, with genome reference Genbank MN908947.3. For a complete analysis and lineage determination, we employed two websites: Nextclade (https://clades.nextstrain.org/, 
accessed on 10 May 2021) and pangolineage (https://pangolin.cog-uk.io/, accessed on 10 May 2021).

\section{Results}

\subsection{Prevalence of SARS-CoV-2 Variants}

Over the studied period, 2207 samples were positive for SARS-CoV-2 from the same number of patients. Using the ID Solution kit screening, $71.8 \%$ of the samples $(1585 / 2207)$ belonged to UK variant (B.1.1.7) (detection of the three targets SARS-CoV-2, N501Y and $\Delta \mathrm{H} 69 / \Delta \mathrm{V} 70$, Figure $1 \mathrm{~A}$ ), and $4.1 \%$ (92 samples/2207) to variants B.1.351 or P.1 (detection of two targets SARS-CoV and 501Y, Figure 1C). The 530 remaining samples $(24.1 \%)$ were other variants, without mutation N501Y nor deletion $\Delta \mathrm{H} 69 / \Delta \mathrm{V} 70$ (e.g., lineages B1.177 or B1.160).

NGS was performed on $67.4 \%(62 / 92)$ of these samples that were suspected to belong to either B.1.351 or P.1 variants. The other 30 samples were not analysed because they were either included in intra-family or intra-institution clusters contaminations (13 cases/30), or harboured a $\mathrm{Ct} \geq 30$ cycles ( 17 cases $/ 30$ ).

After sequencing, $75.8 \%$ of the samples $(47 / 62)$ belonged to lineage B.1.351, and no lineage P.1 was found. Among the 15 remaining samples, surprisingly, 13 were UK variants (B.1.1.7) and 2 belonged to lineage A.27 (19B/501Y) with a N501Y mutation. All of these $13 \mathrm{UK}$ variants presented with one mutation in the S-gene, near the $\Delta \mathrm{H} 69 / \Delta \mathrm{V} 70$ deletion: either S71F in position 21,774, or A67S in position 21,761 on the reference genome. These mutations were present in more than $99 \%$ of all samples (Table 1).
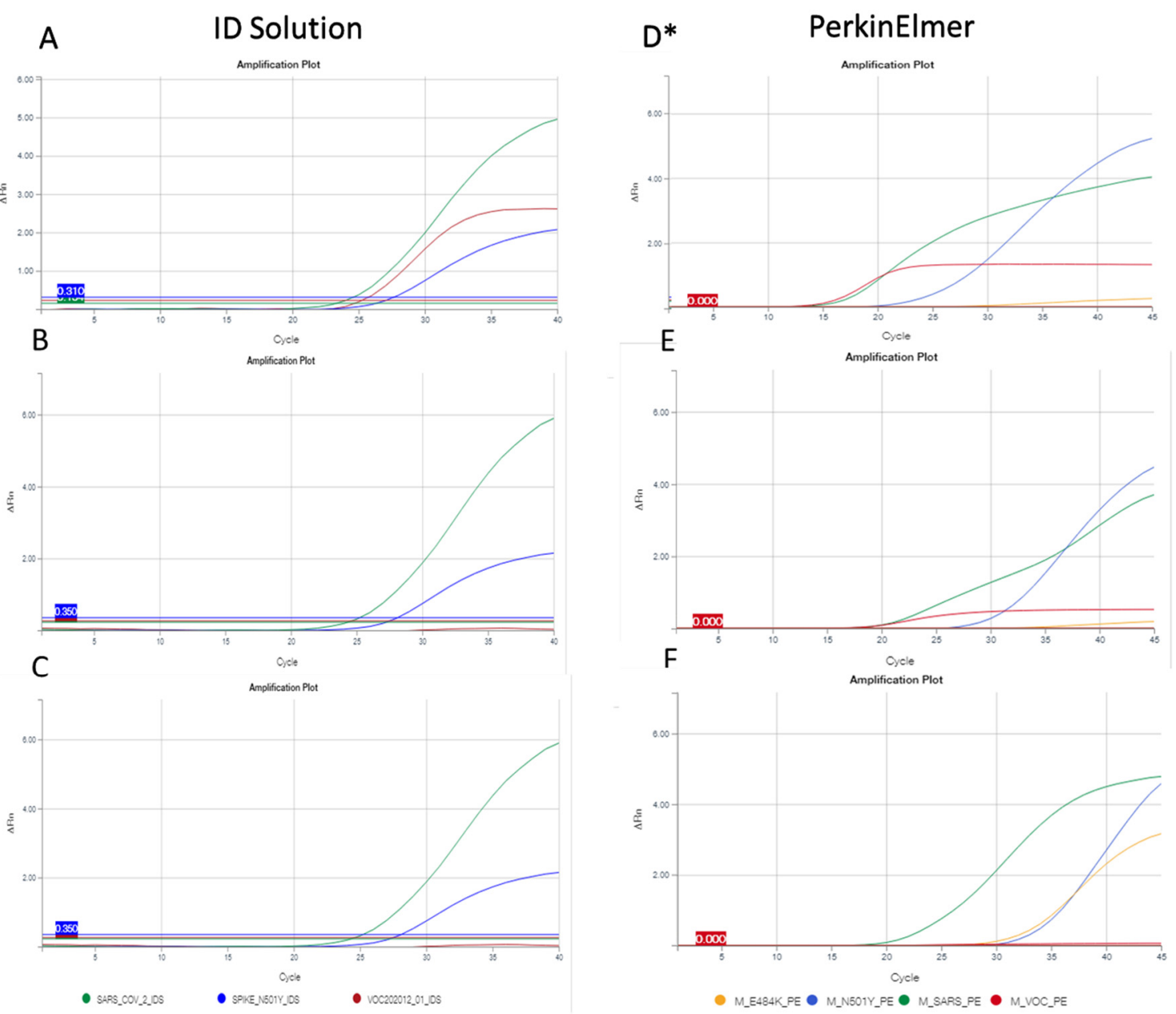

Figure 1. Cont. 


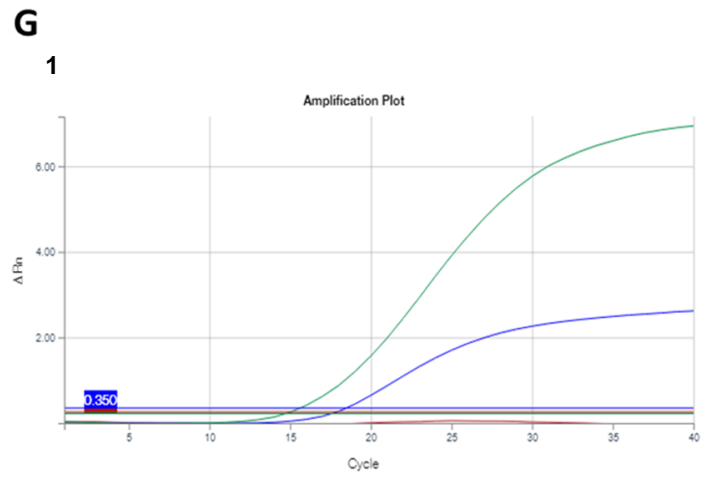

2
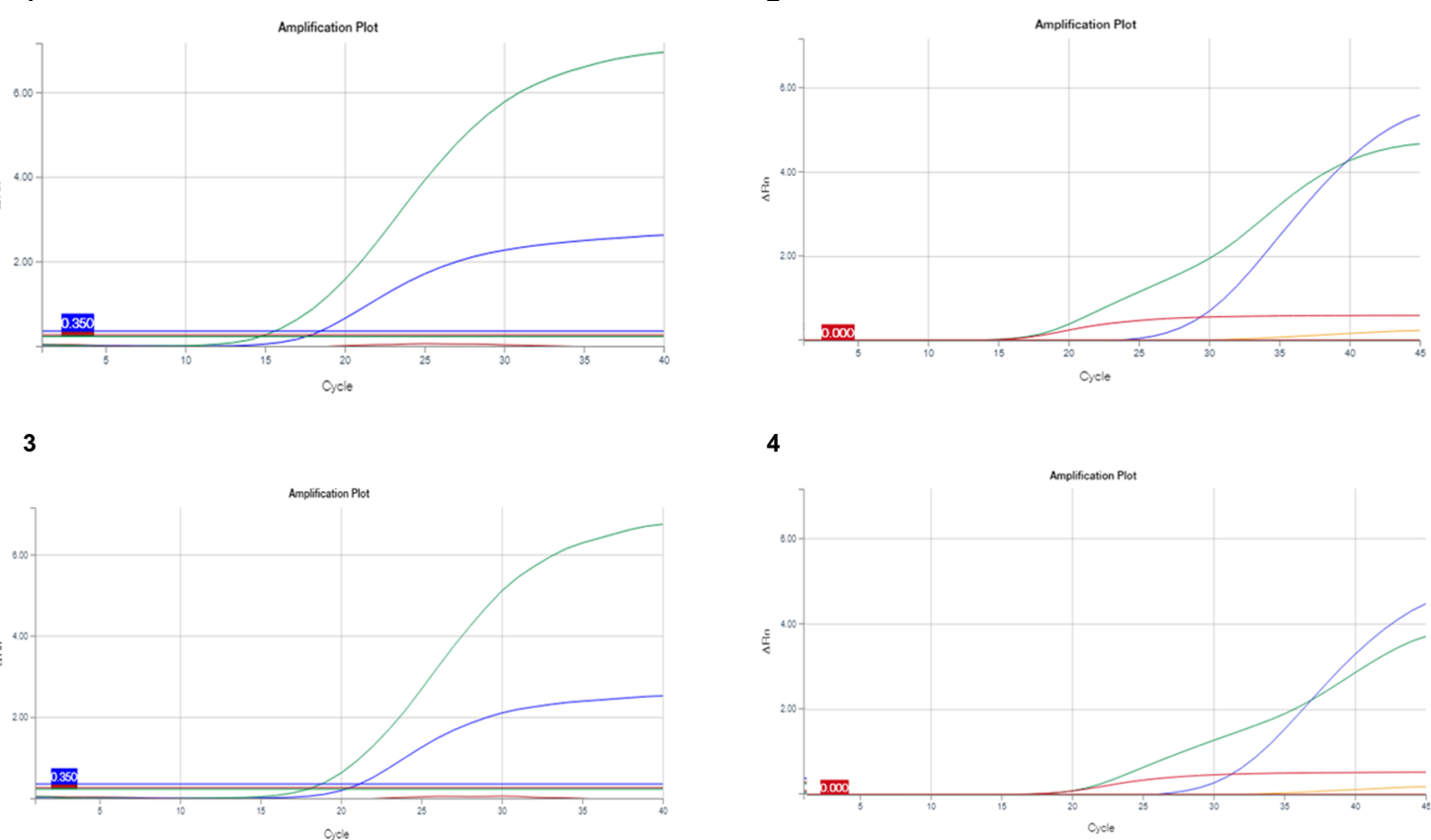

4

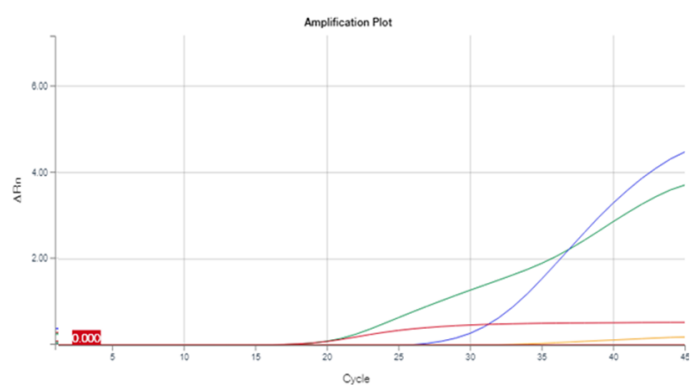

5

6


7

8
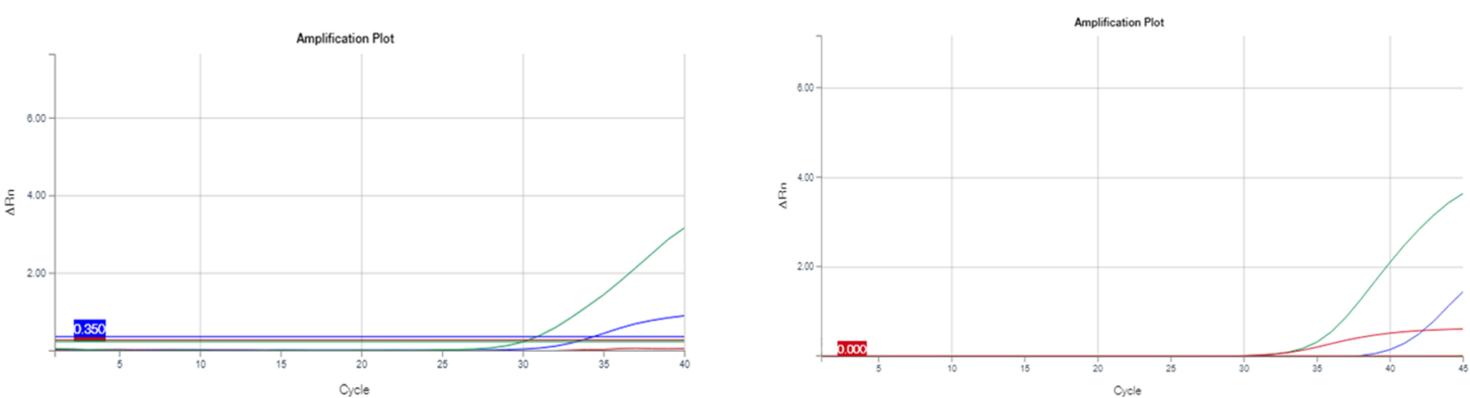

9

10
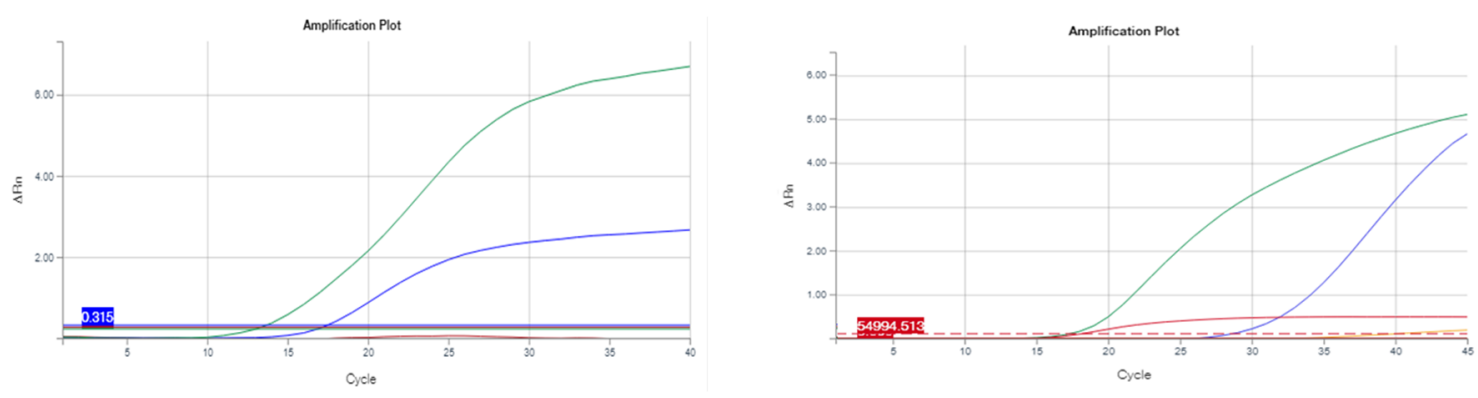

Figure 1. Cont. 

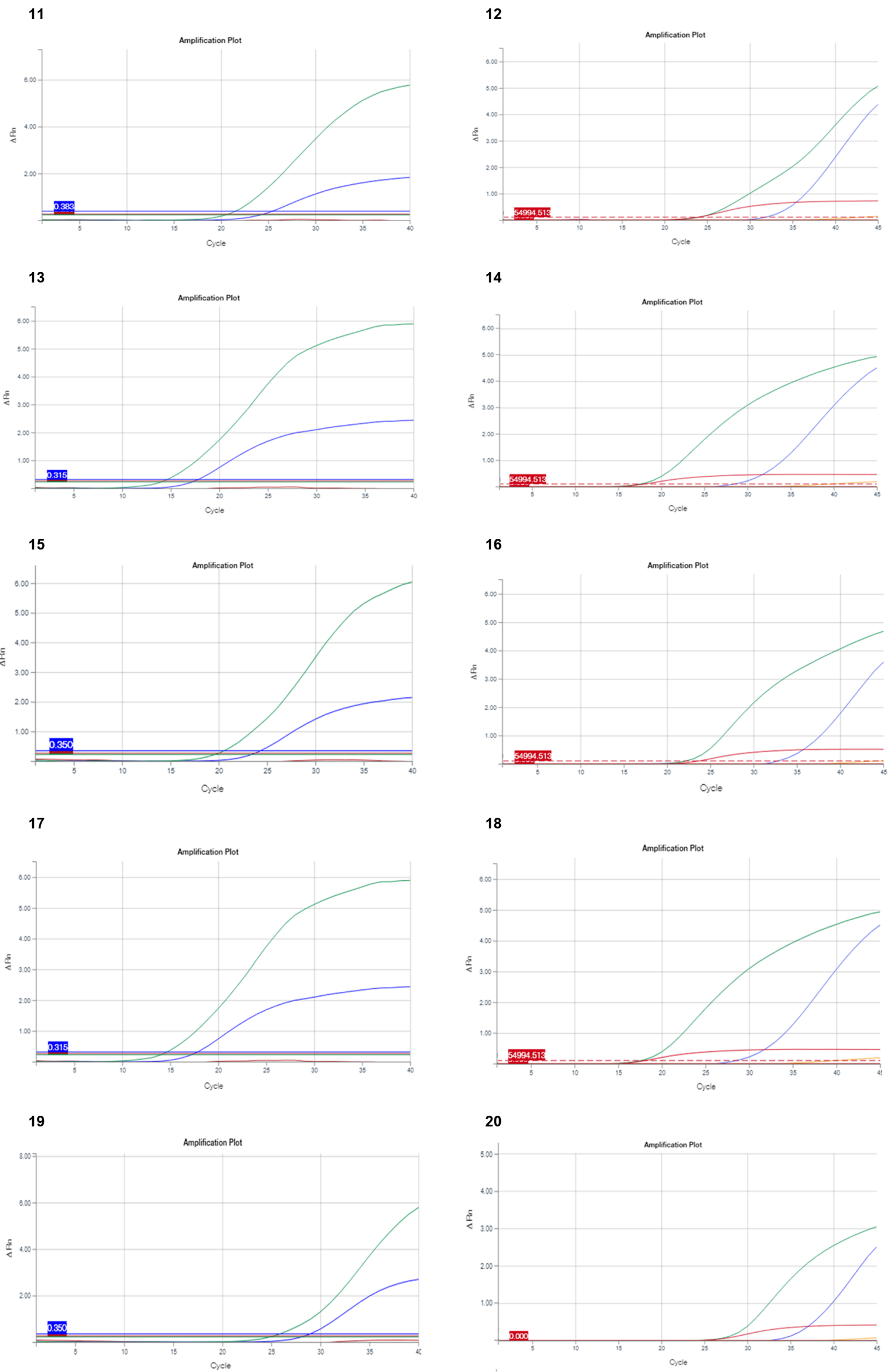

Figure 1. Cont. 
21

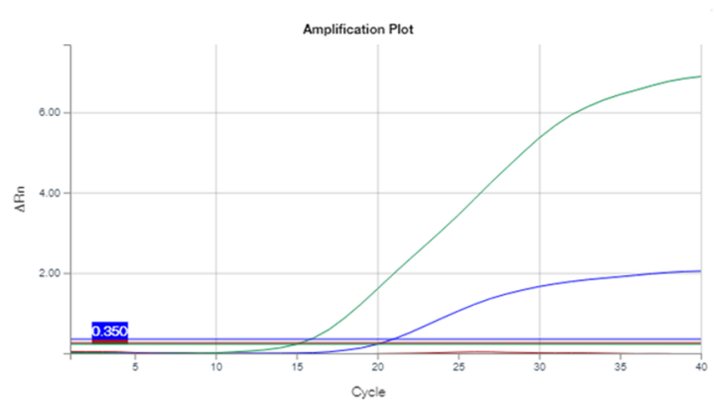

23

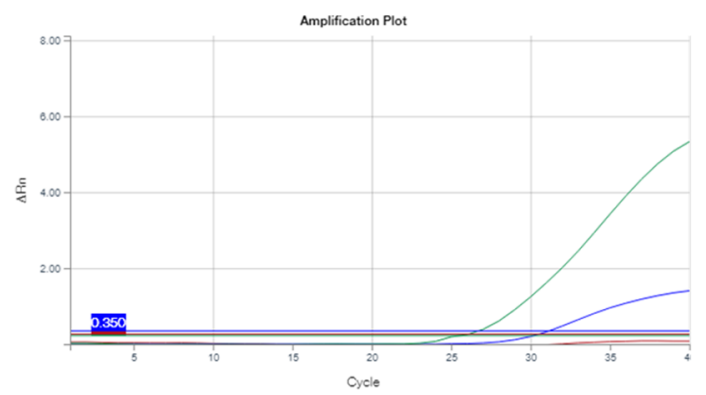

25

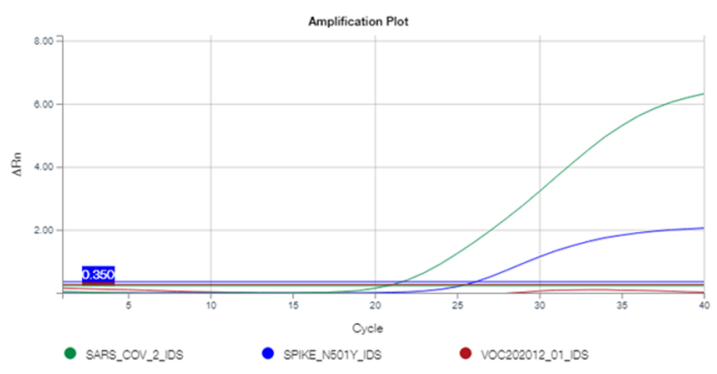

22

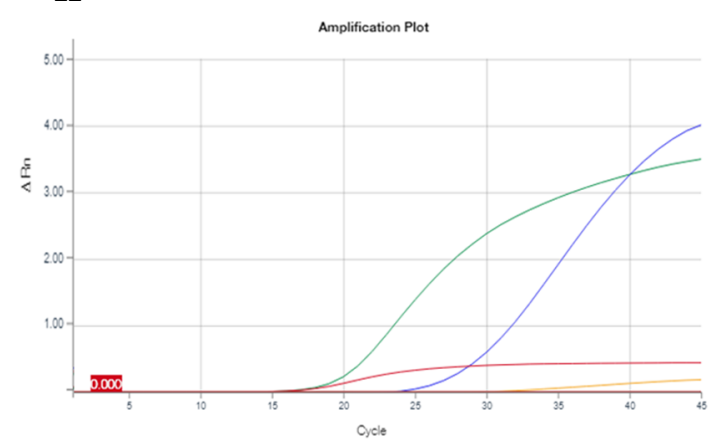

24

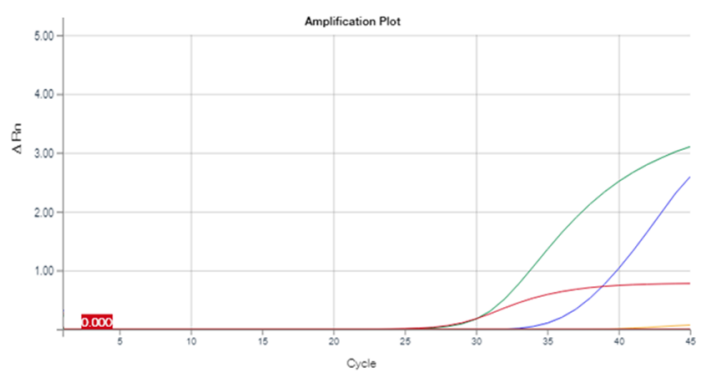

26

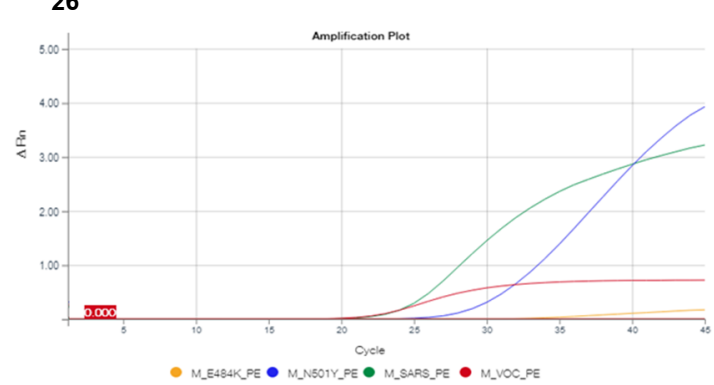

Figure 1. Representation of different variant profiles with the screening RT-PCR assays (on the left the results obtained with the ID Solution kit, and on the right those with PerkinElmer kit). All interpretations of described profiles were derived from NGS analysis. With the ID Solution kit, the green, blue and red curves correspond to SARS-CoV-2, N501Y and $\Delta$ H69/ $\Delta$ V70, respectively. With the PerkinElmer kit, the green, blue, red and orange curves correspond to SARS-CoV-2, N501Y, $\Delta \mathrm{H} 69 / \Delta V 7$ and E484K, respectively. (A,D) profile of B.1.1.7 UK variant; $(\mathbf{B}, \mathbf{E})$ profile of B.1.1.7 variant with S71F mutation; $(\mathbf{C}, \mathbf{F})$ profile of P.1.351 or P.1 variants. * with or without S67A mutation, $(\mathbf{D}, \mathbf{E})$ were B.1.1.7 without E484K mutation. (G) detailed profiles of the 13 samples: 1 and 2: hCoV-19/France/OCC-NI-2104021309/2021; 3 and 4: hCoV-19/France/OCC-NI-2104041142/2021; 5 and 6: hCoV-19/France/OCC-NI-2103280925/2021; 7 and 8: hCoV-19/France/OCC-NI-2104011273/2021; 9 and 10: hCoV-19/France/OCC-NI-2103271225/2021; 11 and 12: hCoV-19/France/OCC-NI-2103301082/2021; 13 and 14: hCoV-19/France/OCC-NI-2103281047/2021; 15 and 16: hCoV-19/France/OCC-NI-2104091053/2021; 17 and 18: hCoV19/France/OCC-NI-2103222347/2021; 19 and 20: hCoV-19/France/OCC-NI-2104270876/2021; 21 and 22: hCoV19/France/OCC-NI-2104290828/2021; 23 and 24: hCoV-19/France/OCC-NI-2104262513/2021; 25 and 26: hCoV19/France/OCC-NI-2104241354/2021. 
Table 1. Details of the characteristics of the NGS sequences deposited in GISAID (Global Initiative on Sharing All Influenza Data).

\begin{tabular}{|c|c|c|c|c|}
\hline Mutation & Virus Name Gisaid & Number GISAID & Coverage & Coverage $(\%)$ \\
\hline \multirow{11}{*}{$\mathrm{S} 71 \mathrm{~F}$} & hCoV-19/France/OCC-NI-2104021309/2021 & EPI_ISL_1972901 & 5835 & $5179 / 5179(100 \%)$ \\
\hline & hCoV-19/France/OCC-NI-2104041142/2021 & EPI_ISL_1972902 & 5867 & $1558 / 1559(99.9 \%)$ \\
\hline & hCoV-19/France/OCC-NI-2103280925/2021 & EPI_ISL_1972903 & 3039 & $1879 / 1882(99.9 \%)$ \\
\hline & hCoV-19/France/OCC-NI-2104011273/2021 & EPI_ISL_1915508 & 4574 & $3247 / 3250(99.9 \%)$ \\
\hline & hCoV-19/France/OCC-NI-2103271225/2021 & EPI_ISL_1915509 & 6630 & $6049 / 6060(99.8 \%)$ \\
\hline & hCoV-19/France/OCC-NI-2103301082/2021 & EPI_ISL_1972904 & 5392 & $2602 / 2604(99.9 \%)$ \\
\hline & hCoV-19/France/OCC-NI-2103281047/2021 & EPI_ISL_1972905 & 2468 & $1737 / 1740(99.9 \%)$ \\
\hline & hCoV-19/France/OCC-NI-2104091053/2021 & EPI_ISL_1915510 & 7209 & $6393 / 6398(99.9 \%)$ \\
\hline & hCoV-19/France/OCC-NI-2103222347/2021 & EPI_ISL_1524908 & 3770 & $3268 / 3269(99.9 \%)$ \\
\hline & hCoV-19/France/OCC-NI-2104270876/2021 & EPI_ISL_2131449 & 5376 & $5866 / 5873(99.9 \%)$ \\
\hline & hCoV-19/France/OCC-NI-2104290828/2021 & EPI_ISL_2131450 & 7257 & $6255 / 6258(99.9 \%)$ \\
\hline \multirow{2}{*}{ A67S } & hCoV-19/France/OCC-NI-2104262513/2021 & EPI_ISL_2131448 & 4244 & $5229 / 5242(99.7 \%)$ \\
\hline & hCoV-19/France/OCC-NI-2104241354/2021 & EPI_ISL_2131447 & 3690 & $3123 / 3126(99.9 \%)$ \\
\hline
\end{tabular}

\subsection{Problems in the Detection of Variants Using Multiplex RT-PCR}

Using the ID Solution kit, the S71F and A67S mutations impacted the detection of $\Delta \mathrm{H} 69 / \Delta \mathrm{V} 70$ deletion (Figure $1 \mathrm{~B}$ ). In all cases, NGS had definitively concluded that the samples belonged to the lineage B.1.1.7 (UK variant).

To investigate if another screening kit had the same problems in the detection of the UK variant, we performed the same screening on the 15 misidentified variants using the PerkinElmer kit. For samples with the S71F mutation, the fluorescence intensity of the $\Delta \mathrm{H} 69 / \Delta \mathrm{V} 70$ target was lower (Figure 1E, red curve) than in B.1.1.7, without this mutation (Figure 1D). In the case of A67S mutations, we did not observe any impact on the detection of the UK variant (Figure 1D). In total, the PerkinElmer kit allowed for the identification of the UK variant, although the fluorescence was not equivalent to the positive control or the UK variant without the mutation S71F.

\section{Discussion}

The consequence of the pandemic SARS-CoV-2 is of concern, due to its high infectivity and fatality rate [16]. The RT-PCR screening that is performed on nasopharyngeal samples is a rapid and inexpensive strategy to characterize the main SARS-CoV-2 variants in a surveillance program to control the virus. This screening was mandatory in France since January 2021. Its implementation in numerous routine labs was simple, compared to that of full-genome sequencing, and has been largely deployed [13]. In our study, we have evaluated the commercialized screening kit that is mainly utilized. The ID Solution kit, performed prospectively in routine, has quickly highlighted the predominance of the UK variant, the most frequent variant currently isolated in France and Europe [13,17,18].

When the RT-PCR using the ID Solution kit detected two targets (universal SARSCoV-2 (N-gene) and N501Y (S-gene)), the manufacturer proposed to conclude that there is the presence of lineage B.1.351; however, the N501Y mutation is present in many variants (e.g., lineage A.27 [19]). Our study supports the interest in performing a full-genome sequencing in these cases. Indeed, among the 62 cases of suspected lineage B.1.351 or P.1, we confirmed the misidentification of 15 samples (24.2\%) that belonged to lineages B.1.1.7 $(21 \%)$ and A.27 (3.2\%). Among these samples, we failed to detect two interesting samples belonging to the UK variant (B.1.1.7). Indeed, the SARS-CoV-2 genome sequencing of these samples highlighted the presence of two mutations, S71F and A67S, localized very closely to the targeted deletion (Figure 2), which could explain the lack of detection. Using the PerkinElmer kit, we observed a low fluorescence intensity in this targeted deletion (Figure 1E), but only in samples with the S71F mutation. The difference between the two screening kits used in this study was the hybridization temperature. This temperature was lower with the PerkinElmer kit $\left(62^{\circ} \mathrm{C}\right)$ than with the ID Solution kit $\left(65^{\circ} \mathrm{C}\right)$. Thus, 
we suspect that the PerkinElmer probes can bind more easily to the sequence genome, despite the presence of the S71F mutation. However, this binding was hindered by the mutation and the amplification curve, which corresponded to a weaker fluorescence signal than expected. Moreover, we can hypothesize that the sequences of the PCR primers are different, even if they are not known by users, as we can detect the A67S mutation with the PerkinElmer kit. Our results suggest that a heightened attention of the virologist is needed during the result interpretation.


Figure 2. CLC genomics view of two mutations near the deletion $\Delta H 69 / \Delta V 70$. (A) mutation view of S71F; (B) mutation view of A67S.

Recent studies have evaluated different screening kits in comparison to NGS. The authors demonstrated a high concordance between the two methods, reinforcing the interest of screening methods [20-24]. Here, we showed that the PerkinElmer kit performed better than the ID Solution kit, avoiding the problem of mutation detection, notably the detection of E484K mutation. This mutation is always present in the lineages B.1.351 and P.1, but also in the UK variant [25]. The detection of the E484K mutation has a clinical importance because it is associated with a reduced efficacy of the vaccine, or confers resistance to monoclonal antibodies or convalescent plasma [10-12]. However, even if the PerkinElmer kit presented better performance in our study, we suggest that a similar problem of detection could be observed if a mutation was localized closed to the E484K target, impacting on the two RT-PCR screening kits assays. Given the number of mutations present in the different VOCs and the constant appearance of new variants of interest (VOI), we assume that these screenings methods targeting few mutations are less adapted to the evolution of the COVID-19 epidemic [25].

Currently, despite the obligation of Direction Générale de la Santé (DGS) in France, the screening of the UK variant, in view of this prevalence, is no longer necessary for diagnosis or clinical impact [26]. With the evolution of VOCs and VOIs, the French recommendations evolved on 26 May 2021 to screen only E484K and two other mutations (E484Q, L452R), with an impact similar to E484K. It is important to know that rapid screening tools seem limited to follow the evolution of the virus and detect the different mutations $[8,25,27]$. Although NGS is more expensive, time consuming, and needs adapted structures with 
technical and biological experience, this technology is the unique tool able to determine the different variants and follow the evolution of SARS-CoV-2.

Author Contributions: Conceptualization, A.B. and R.S.; methodology, A.B., R.S., S.B., M.S. and J.-P.L.; software, A.B. and S.B.; validation, R.S., M.S. and J.-P.L.; formal analysis, A.B., R.S., S.B. and M.S.; investigation, A.B.; resources, J.-P.L.; data curation, A.B., R.S., S.B., M.S. and J.-P.L.; writing - original draft preparation, A.B. and J.-P.L.; writing—review and editing, R.S., S.B. and M.S.; visualization, A.B., R.S. and M.S.; supervision, J.-P.L.; project administration, A.B. and J.-P.L.; funding acquisition, J.-P.L. All authors have read and agreed to the published version of the manuscript.

Funding: This research received no external funding.

Institutional Review Board Statement: The study was conducted according to the guidelines of the Declaration of Helsinki, and approved by the Institutional Review Board of Nîmes University Hospital (IRB number: 20.05.01, approved on 4 May 2020).

Informed Consent Statement: Non-opposed consent was obtained from all subjects involved in the study.

Data Availability Statement: Not applicable.

Acknowledgments: We thank Sarah Kabani for her editing assistance. We thank the Nîmes University hospital for its structural, human, and financial support through the award obtained by our team during the internal call for tenders "Thématiques phares". The authors belong to the FHU InCh (Federation Hospitalo Universitaire Infections Chroniques, Aviesan).

Conflicts of Interest: The authors declare no conflict of interest.

\section{References}

1. Zhu, N.; Zhang, D.; Wang, W.; Li, X.; Yang, B.; Song, J.; Zhao, X.; Huang, B.; Shi, W.; Lu, R.; et al. A novel coronavirus from patients with pneumonia in China, 2019. N. Engl. J. Med. 2020, 382, 727-733. [CrossRef]

2. Korber, B.; Fischer, W.M.; Gnanakaran, S.; Yoon, H.; Theiler, J.; Abfalterer, W.; Hengartner, N.; Giorgi, E.E.; Bhattacharya, T.; Foley, B.; et al. Tracking changes in SARS-CoV-2 spike: Evidence that D614G increases infectivity of the COVID-19 virus. Cell 2020, 182, 812-827.e19. [CrossRef] [PubMed]

3. Preliminary Genomic Characterisation of an Emergent SARS-CoV-2 Lineage in the UK Defined by a Novel Set of Spike Mutations. Available online: https:/ / virological.org/t/preliminary-genomic-characterisation-of-an-emergent-sars-cov-2-lineage-in-theuk-defined-by-a-novel-set-of-spike-mutations/563 (accessed on 30 May 2021).

4. Rambaut, A.; Holmes, E.C.; O’Toole, Á.; Hill, V.; McCrone, J.T.; Ruis, C.; du Plessus, L.; Pybus, O.G. A dynamic nomenclature proposal for SARS-CoV-2 lineages to assist genomic epidemiology. Nat. Microbiol. 2021, 6, 415. [CrossRef] [PubMed]

5. Tegally, H.; Wilkinson, E.; Lessells, R.J.; Giandhari, J.; Pillay, S.; Msomi, N.; Mlisana, K.; Bhiman, J.N.; von Gottberg, A.; Walaza, S.; et al. Sixteen novel lineages of SARS-CoV-2 in South Africa. Nat. Med. 2021, 27, 440-446. [CrossRef]

6. Faria, N.R.; Mellan, T.A.; Whittaker, C.; Claro, I.M.; Candido, D.D.S.; Mishra, S.; Crispim, M.A.E.; Sales, F.C.S.; Hawryluk, I.; McCrone, J.T.; et al. Genomics and epidemiology of the P.1 SARS-CoV-2 lineage in Manaus, Brazil. Science 2021, 372, 815-821. [CrossRef]

7. Galloway, S.E.; Paul, P.; MacCannell, D.R.; Johansson, M.A.; Brooks, J.T.; MacNeil, A.; Slayto, R.B.; Tong, S.; Silk, B.J.; Armstrong, G.L.; et al. Emergence of SARS-CoV-2 B.1.1.7 lineage-United States, December 29, 2020-January 12, 2021. Morb. Mortal. Wkly. Rep. 2021, 70, 95-99. [CrossRef]

8. European Centre for Disease Prevention and Control. SARS-CoV-2 Variants of Concern as of 3 June 2021. Available online: https: / / www.ecdc.europa.eu/en/covid-19/variants-concern (accessed on 30 May 2021).

9. Bal, A.; Destras, G.; Gaymard, A.; Stefic, K.; Marlet, J.; Eymieux, S.; Regue, H.; Semanas, Q.; d'Aubarede, C.; Billaud, G.; et al. Two-step strategy for the identification of SARS-CoV-2 variant of concern 202012/01 and other variants with spike deletion H69-V70, France, August to December 2020. Eurosurveillance 2021, 26, 2100008. [CrossRef]

10. Weisblum, Y.; Schmidt, F.; Zhang, F.; DaSilva, J.; Poston, D.; Lorenzi, J.C.; Muecksch, F.; Rutkowska, M.; Hoffmann, H.H.; Michailidis, E.; et al. Escape from neutralizing antibodies by SARS-CoV-2 spike protein variants. ELife 2020, 9, e61312. [CrossRef] [PubMed]

11. Zhou, D.; Dejnirattisai, W.; Supasa, P.; Liu, C.; Mentzer, A.J.; Ginn, H.M.; Zhao, Y.; Duyvesteyn, H.M.E.; Tuekprakhon, A.; Nutalai, R.; et al. Evidence of escape of SARS-CoV-2 variant B.1.351 from natural and vaccine-induced sera. Cell 2021, 184, 2348-2361.e6. [CrossRef] [PubMed]

12. Wu, K.; Werner, A.P.; Koch, M.; Choi, A.; Narayanan, E.; Stewart-Jones, G.B.E.; Colpitts, T.; Bennett, H.; Boyoglu-Barnum, S.; Shi, W.; et al. Serum neutralizing activity elicited by mRNA-1273 vaccine. N. Engl. J. Med. 2021, 384, 1468-1470. [CrossRef] [PubMed] 
13. Haim-Boukobza, S.; Roquebert, B.; Trombert-Paolantoni, S.; Lecorche, E.; Verdurme, L.; Foulongne, V.; Selinger, C.; Michalakis, Y.; Sofonea, M.T.; Alizon, S. Detecting rapid spread of SARS-CoV-2 variants, France, January 26-February 16, 2021. Emerg. Infect. Dis. 2021, 27, 1496-1499. [CrossRef]

14. Yan, Y.; Chang, L.; Luo, W.; Liu, J.; Guo, F.; Wang, L. Comparison of seven commercial severe acute respiratory syndrome coronavirus 2 nucleic acid detection reagents with pseudovirus as quality control material. J. Mol. Diagn. 2021, 23, 300-309. [CrossRef] [PubMed]

15. Ion AmpliSeq ${ }^{\mathrm{TM}}$ Library Kit Plus USER GUIDE. Available online: http://tools.thermofisher.com/content/sfs/manuals/MAN0 017003_IonAmpliSeqLibaryKitPlus_UG.pdf (accessed on 30 May 2021).

16. Wiersinga, W.J.; Rhodes, A.; Cheng, A.C.; Peacock, S.J.; Prescott, H.C. Pathophysiology, transmission, diagnosis, and treatment of coronavirus disease 2019 (COVID-19): A review. JAMA 2020, 324, 782-793. [CrossRef] [PubMed]

17. Area, I.; Nieto, J.J. Forecasting the effects of the new SARS-CoV-2 variant in Europe. Sci. World J. 2021, 2021, 5553240. [CrossRef] [PubMed]

18. Funk, T.; Pharris, A.; Spiteri, G.; Bundle, N.; Melidou, A.; Carr, M.; Gonzalez, G.; Garcia-Leon, A.; Crispie, F.; O'Connor, L.; et al. Characteristics of SARS-CoV-2 variants of concern B.1.1.7, B.1.351 or P.1: Data from seven EU/EEA countries, weeks $38 / 2020$ to 10/2021. Eurosurveillance 2021, 26, 2100348. [CrossRef] [PubMed]

19. Li, X.; Giorgi, E.E.; Marichannegowda, M.H.; Foley, B.; Xiao, C.; Kong, X.P.; Chen, Y.; Gnanakaran, S.; Korber, B.; Gao, F. Emergence of SARS-CoV-2 through recombination and strong purifying selection. Sci. Adv. 2020, 6, eabb9153. [CrossRef]

20. Kami, W.; Kinjo, T.; Arakaki, W.; Oki, H.; Motooka, D.; Nakamura, S.; Fujita, J. Rapid and simultaneous identification of three mutations by the Novaplex ${ }^{\mathrm{TM}}$ SARS-CoV-2 variants I assay kit. J. Clin. Virol. 2021, 141, 104877. [CrossRef] [PubMed]

21. Vega-Magaña, N.; Sánchez-Sánchez, R.; Hernández-Bello, J.; Venancio-Landeros, A.A.; Peña-Rodríguez, M.; Vega-Zepeda, R.A.; Galindo-Ornelas, B.; Díaz-Sánchez, M.; García-Chagollán, M.; Macedo-Ojeda, G.; et al. RT-qPCR assays for rapid detection of the N501Y, 69-70del, K417N, and E484K SARS-CoV-2 mutations: A screening strategy to identify variants with clinical impact. Front. Cell. Infect. Microbiol. 2021, 11, 672562. [CrossRef] [PubMed]

22. Wang, H.; Miller, J.A.; Verghese, M.; Sibai, M.; Solis, D.; Mfuh, K.O.; Jiang, B.; Iwai, N.; Mar, M.; Huang, C.; et al. Multiplex SARS-CoV-2 genotyping RT-PCR for population-level variant screening and epidemiologic surveillance. J. Clin. Microbiol. 2021, JCM0085921. [CrossRef]

23. Amato, L.; Jurisic, L.; Puglia, I.; Di Lollo, V.; Curini, V.; Torzi, G.; Girolamo, A.D.; Mangone, I.; Mancinelli, A.; Decaro, N.; et al. Multiple detection and spread of novel strains of the SARS-CoV-2 B.1.177 (B.1.177.75) lineage that test negative by a commercially available nucleocapsid gene real-time RT-PCR. Emerg. Microbes. Infect. 2021, 10, 1148-1155. [CrossRef] [PubMed]

24. Wang, H.; Jean, S.; Eltringham, R.; Madison, J.; Snyder, P.; Tu, H.; Jones, D.M.; Leber, A.L. Mutation-specific SARS-CoV-2 PCR screen: Rapid and accurate detection of variants of concern and the identification of a newly emerging variant with spike L452R mutation. J. Clin. Microbiol. 2021, JCM-00926. [CrossRef]

25. SARS-CoV-2 Variant Classifications and Definitions. Available online: https://www.cdc.gov/coronavirus/2019-ncov/variants/ variant-info.html (accessed on 30 May 2021).

26. DGS-URGENT N 2021_12: Stratégie de Freinage de la Propagation des Variants du SARS-CoV-2: Renforcement Spécifique sur les Variants d'Interet 20H/501Y.V2 et 20J/501Y.V3. Available online: https:/ / solidarites-sante.gouv.fr/ministere/declarationspubliques-d-interets / direction-generale-de-la-sante-dgs (accessed on 30 May 2021).

27. Verghese, M.; Jiang, B.; Iwai, N.; Mar, M.; Sahoo, M.K.; Yamamoto, F.; Mfuh, K.O.; Miller, J.; Wang, H.; Zehnder, J.; et al. A SARS-CoV-2 Variant with L452R and E484Q Neutralization Resistance Mutations. J. Clin. Microbiol. 2021, 59, e0074121. [CrossRef] [PubMed] 